\title{
Experimental Study on the Coupled Heat-Moisture-Heavy Metal Pollutant Transfer Process in Soils
}

\author{
Qingke Nie ${ }^{\mathbb{D}},{ }^{1,2}$ Wei Wang, ${ }^{1}$ Wenkai Guo, ${ }^{3}$ and Huawei $\mathrm{Li}^{1,2}$ \\ ${ }^{1}$ Hebei Research Institute of Construction and Geotechnical Investigation Co. Ltd., Shijiazhuang 050031, China \\ ${ }^{2}$ Center for Geotechnical Engineering Technology of Hebei Province, Shijiazhuang 050031, China \\ ${ }^{3}$ School of Civil Engineering, Beijing Jiaotong University, Beijing 100044, China \\ Correspondence should be addressed to Qingke Nie; niealex@126.com
}

Received 1 March 2021; Revised 14 March 2021; Accepted 22 March 2021; Published 29 March 2021

Academic Editor: Faming Huang

Copyright ( 92021 Qingke Nie et al. This is an open access article distributed under the Creative Commons Attribution License, which permits unrestricted use, distribution, and reproduction in any medium, provided the original work is properly cited.

\begin{abstract}
The coupled physical mechanism of heat conduction, moisture migration, and heavy metal transfer in a kaolin soil was studied by one-dimensional column tests. Two cyclic temperature tests show that, during the second cycle, the temperature close to the heat source of the soil column is lower than that during the first cycle and the temperature far away from the heat source is low, which reflects the influence of heating path. Correspondingly, the moisture content distribution during the second cycle is quite different from that during the first cycle. The higher the soil dry density is, the better the heat conduction is. The lower the dry density is, the more favorable the moisture migration is. The placement direction of the soil column and the set of temperature boundaries affect the moisture distribution of the soil column through the difference in the temperature, gravity, and solid matrix potentials. The temperature-driven liquid water movement effectively promotes the transfer of heavy metal contaminant in unsaturated soils; it is closely correlated with the convection of the heavy metal substances easily dissolved in liquid water. However, the transfer of heavy metal substances in unsaturated soil is not obvious without a thermal driving force. The test results for the different heavy metal ions indicate that the thermally induced transfer distance of the heavy metal pollutants with low adsorption properties $\left(\right.$ e.g., $\left.\mathrm{Cu}^{2+}\right)$ to soil particles is much larger than that of the heavy metal pollutants with high adsorption properties (e.g., Cd $\left.{ }^{2+}\right)$.
\end{abstract}

\section{Introduction}

Rapid industrial and agricultural production has caused the extensive occurrence of pollutants in soil layers due to contaminant transport, such as organic and inorganic matter and even heavy metal ions [1-3], causing widespread ecosystem damage in environmental engineering $[4,5]$. Among these pollutants, the transfer mechanism and treatment measures of heavy metal contaminants (e.g., $\mathrm{Cd}^{2+}$, $\mathrm{Pb}^{2+}, \mathrm{Cu}^{2+}, \mathrm{Cr}^{2+}$, and $\mathrm{Zn}^{2+}$ ) have become the focus issue of geoenvironmental research [6-8]. The interactions between soil particles and heavy metal contaminants can differ substantially [9-11], which depends on their deposition mechanism and the imposed external conditions [12], including the aspects such as the $\mathrm{pH}$ value and ionic concentration of solutions, van der Waals forces, double layer repulsive force, presence of colloidal particles [13, 14], and even the temperature effect $[15,16]$.
The transfer of contaminants has been largely investigated through experimental research, theoretical analyses, and numerical simulations $[17,18]$. Its application fields include industrial pollutant treatment in groundwater exploitation and recharge, geothermal resource development, and waste disposal in metal mining [19]. Lee et al. [5] discovered that the concentrations of $\mathrm{Cr}^{2+}$ and $\mathrm{Pb}^{2+}$ in soil pores increase with increasing the colloidal concentration; as a result, the release of $\mathrm{Cd}^{2+}$ and $\mathrm{Cu}^{2+}$ lags behind that of colloidal particles. Cui et al. [20] discussed the detachment processes of previously deposited silicon powders in soils and reported that the seepage direction of water is more important than the seepage rate. Monged et al. [21] investigated the differences in the occurrence state, adsorption concentration, migration law, and migration speed of heavy metal contaminants (i.e., $\mathrm{Cd}^{2+}, \mathrm{Cu}^{2+}, \mathrm{Pb}^{2+}, \mathrm{Zn}^{2+}$, and $\mathrm{Ni}^{2+}$ ) located in a geothermal resource development area and nearby strata and evaluated the safety of the polluted strata. 
Overall, the present research on the retardation mechanism and transfer process of contaminants was accomplished mainly considering a hydraulic/chemical gradient [22]. However, the thermally induced transfer of heavy metal contaminants in porous media widely occurs in the protection of buildings, the treatment of metal iron ore, landfill leachate diffusion, thermal energy storage, and nuclear waste disposal. Funk and Wakili [23] proposed a thermodynamic transport model to describe the contaminant transfer induced by temperature. Miracapillo and Ferroni [24] numerically calculated the radionuclide transfer in a geological repository site and analyzed the transfer process of contaminants. Nguyen and Amiri [25] assessed the transfer process of some typical ions (e.g., cation $\mathrm{Na}^{+}$, anion $\mathrm{Cl}^{-}$, and $\mathrm{OH}^{-}$) in soils caused by heating. Tang and Cui [26] elaborated the temperature influence in the deformation of bentonite and investigated the transfer of radioactive matters.

For unsaturated soils, the matric suction and temperature effect are generally interacted, which greatly changes the thermal and hydraulic properties of soils [27, 28]. Heat conduction induces notable changes in the temperature potentials of soils and makes moisture migrate from hightemperature locations to low-temperature locations $[29,30]$. Bai et al. [31] proposed a theoretical model of the soil-water characteristic curve (SWCC) taking into account the influence of water flow as well as thermal conduction using the so-called nonequilibrium thermodynamics theory. Cui et al. [32] studied the desorption process of already adsorbed particles on the surfaces of porous media matrix by laboratory experiments, which showed that both increasing the flow velocity and changing the flow direction alter the detachment rate of the deposited particles. Bai and Shi [33] considered the transition between gas and liquid phases by the temperature and established a relationship for the content of bound water in pores measured by the volume density technique.

In this study, the physical mechanism of heat transfer in unsaturated soil (i.e., kaolin soil) is investigated by column tests, along with the moisture migration and heavy metal transfer. To this end, the changes in temperature and moisture content and the steady-state distribution characteristics are studied, and the influence of the soil dry density, soil column placement direction, and temperature boundary on the heat and moisture transfer process is analyzed. In addition, experiments are also performed to elucidate the temperature influence on the transfer mechanism of heavy metal pollutants. This study has important applications for the evaluation and prediction of environmental impacts.

\section{Materials and Methods}

2.1. Test Apparatus. The test equipment is a hollow cylindrical chamber, which is constructed of organic glass with heat-resisting temperature (Figure 1). The size of the specimen is $L=102 \mathrm{~cm}$ in length and $D=14.8 \mathrm{~cm}$ in the inner diameter, and their ratio is 6.9. Hence, the test soil column is herein considered as a one-dimensional case. This device consists of two cylinders each with a length of $51 \mathrm{~cm}$.
The two parts are fixed by bolts and sealed by rubber rings on their cross sections. The sidewall of the cylinder is covered with thick insulation materials. The heat source of the chamber is connected by a circulating water tank with a controlled constant temperature in the range of $20^{\circ} \mathrm{C}$ to $100^{\circ} \mathrm{C}$ (HH-601 Model, Shanghai city, China). The interface between the soil column end and the heat source is connected with a thin steel plate to guarantee excellent heat conduction. Small holes are made on the cylinder wall to install moisture and temperature sensors. The distances of the holes from the heat source are 2, 7, 17, 27, 42, 57, 72, and $88 \mathrm{~cm}$, respectively.

The temperature is measured with small platinum resistance sensors (PT100 Mode, JY Company, Beijing, China) and a JY-DAM-TC16 collector. The measurement accuracy is $0.5^{\circ} \mathrm{C}$ in the test range of 0 to $100^{\circ} \mathrm{C}$. The moisture content is measured with small capacitive sensors (EC-5 Mode, Decagon Company, USA) and an Em50 collector. The test results of moisture content in the experiment are very sensitive, and careful calibration should be guaranteed.

2.2. Test Materials. Kaolin clay has low permeability and good adsorption property, which has been widely used in environment pollution control in soil layers. The selected kaolin clay utilized in this study was collected from in Hebei Province of China (Lingshou County). A laser diffraction instrument (LA-950 Mode, HORIBA, Ltd., Japan) was used to measure the particle size distribution of the sample (wet method). The median particle size of the sample is $D_{50}=15.7 \mu \mathrm{m}$, and the particle size ranges from 1 to $90 \mu \mathrm{m}$. The soil particle density is $\rho_{\mathrm{s}}=2.56 \mathrm{~g} / \mathrm{cm}^{3}$. The composition of the sample measured by X-ray diffraction produced by Bruker Corporation (D8 Advance Mode, Germany) mainly includes quartz $\left(\mathrm{SiO}_{2}, 35.5 \%\right)$, albite $\left(\mathrm{NaAlSi}_{3} \mathrm{O}_{8}, 47.9 \%\right)$, and sanidine $\left(\mathrm{KNaAlSi}_{3} \mathrm{O}_{8}, 16.6 \%\right)$.

Generally, the adsorption capacity of copper ions $\left(\mathrm{Cu}^{2+}\right)$ to soil particles is much lower than that of cadmium ions $\left(\mathrm{Cd}^{2+}\right)$, which is a typical heavy metal pollutant in geoenvironmental engineering [3,4,34]. For comparison, these two heavy metal ions are selected to examine the temperature-driven transfer process in this paper. Here, copper nitrate solution (i.e., $\left.\mathrm{Cu}\left(\mathrm{NO}_{3}\right)_{2}\right)$ and cadmium nitrate solution (i.e., $\mathrm{Cd}\left(\mathrm{NO}_{3}\right)_{2}$ ) were employed as heavy metal contaminants. Disodium ethylenediaminetetraacetate (EDTA-2Na) with high solubility was used as an extractant to separate the $\mathrm{Cu}^{2+}$ and $\mathrm{Cd}^{2+}$ ions adsorbed onto the soil particles [7, 11, 35].

2.3. Test Procedures. Soil samples with specified moisture content were prepared at an ambient temperature of $T=22^{\circ} \mathrm{C}$ for laboratory testing. For the convenience of operation, the test cylinder is placed vertically, and afterwards, the soil material is filled in layers. The filling is divided into 20 layers, so the thickness of each layer is about $5 \mathrm{~cm}$. During the filling process, the corresponding sensors should be carefully placed in the predetermined position and compacted with soil. Then, the sensor wire is led out through the preset cylinder wall hole and connected with the data acquisition system (Figure 1). 


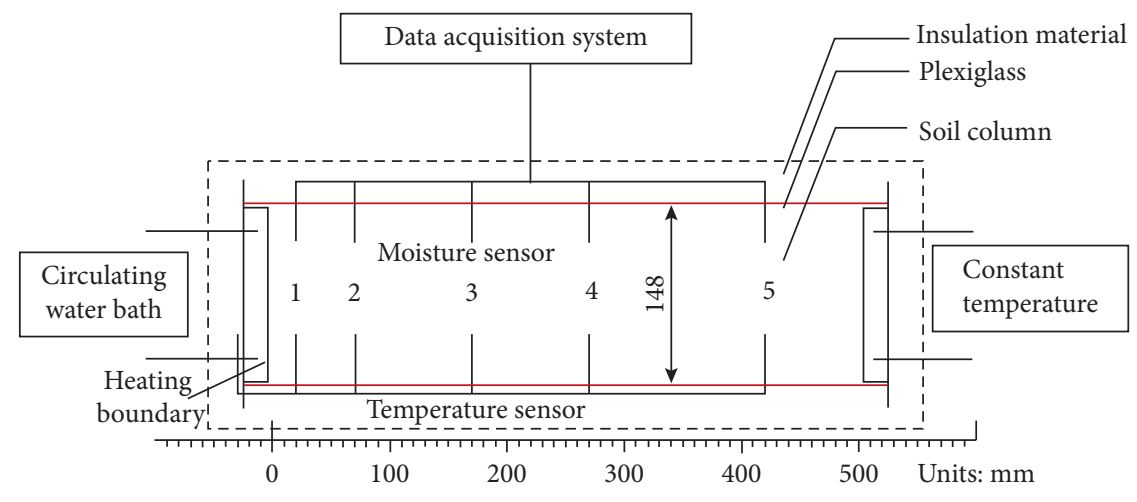

Figure 1: Experimental apparatus (half of the length).

During the test, the heat loss of the cylinder sidewall cannot be ignored. In order to correct the temperature boundary, the corresponding temperature at the sidewall and both ends of the cylinder are also measured.

Moisture migration tests of the soil column under cyclic temperature loading are executed, including 5 test schemes (Table 1). The initial mass moisture content of the specimen is set to $18.0 \%$, including three different initial dry densities (i.e., $\rho_{\mathrm{d}}=1.4,1.3$, and $1.2 \mathrm{~g} / \mathrm{cm}^{3}$ ) and two placement directions of the soil columns (i.e., horizontal and vertical with different heating ends). Moreover, the initial volume moisture content and saturation degree are different, as summarized in Table 1, where the volume moisture content refers to the ratio of the volume occupied by moisture to the soil column volume. The temperature at the heating end is controlled at approximately $73^{\circ} \mathrm{C}$ during the heating stage (Figure 1 ) or $25^{\circ} \mathrm{C}$ during the cooling stage. In fact, the control of boundary temperature is very sensitive due to the heat dissipation effect. The temperature range herein is given by the actual measured value of the tests. In addition, the low-temperature end of the soil column is always maintained at $22^{\circ} \mathrm{C}$.

The transfer of heavy metal ions $\mathrm{Cu}^{2+}$ and $\mathrm{Cd}^{2+}$ caused by heating is investigated. Therefore, a certain thickness of contaminated soil (i.e., pollution source, and thickness of $5 \mathrm{~mm}$ ) should be filled near the heating end of the cylinder. The standard copper nitrate $\left(\mathrm{Cu}\left(\mathrm{NO}_{3}\right)_{2}\right)$ or lead nitrate $\left(\mathrm{Cd}\left(\mathrm{NO}_{3}\right)_{2}\right)$ solutions were prepared to the required concentration and then mixed with the kaolin sample of a certain quality. Then, according to the filling method and density requirements similar to the previous clean soil sample, the pollutant soil layer is filled. Finally, the concentration of pollution source $\left(C_{0}\right)$ was calculated (Table 2) for the subsequent evaluation of the pollutant migration process. For convenience, the concentration of the pollution source refers to the mass of heavy metal pollutants in the water contained in unit mass soil particles.

In these tests, the soil column is placed horizontally. The location of the heating end is set to the left end except for case 9 (no thermal driving force). The temperature of the heat source can remain at $73^{\circ} \mathrm{C}$. Thus, the moisture content is set to $\theta_{0}=18.0 \%$ in the initial state, and the measured $\mathrm{Cu}^{2+}$ and $\mathrm{Cd}^{2+}$ concentrations in the solution are $C_{0}=1 \mathrm{mg} / \mathrm{g}$.

In the above transfer tests of heavy metal ions, when the heating experiment is completed, the samples are taken at different positions of the test soil column to determine the concentration of copper $\left(\mathrm{Cu}^{2+}\right)$ or lead ions $\left(\mathrm{Pb}^{2+}\right)$ in the contaminated soil caused by temperature. Three soil samples at the same section of the soil column are taken out for parallel measurement, and finally, their average values were adopted. For this, a small amount of soil samples (about $3 \mathrm{~g}$ ) was dried, and $0.15 \mathrm{~mol} / \mathrm{L}$ EDTA solution was added to extract the heavy metal ions adsorbed on the soil particles. The mixed solution is placed on the oscillator for vibration (HZQ-F160, Shanghai city, China) and centrifuged using a high-speed centrifuge (Sigma 3K15, Germany). Then, the supernatant is taken out for the measurement of the concentration of heavy metal substances which was determined by a graphite furnace spectrophotometer (TAS-990G Mode, Puxi Co., China) [36,37].

\section{Results and Discussion}

3.1. Temperature Evolution Process. As a typical cyclic heating process, case 1 (Table 1) is selected as an example to illustrate the evolution process of the temperature over time at different distances of the soil column under the action of cyclic temperature loading (two cycles). Figure 2 shows that, during the heating stage, the temperature at the measuring points close to the heat source (i.e., at $x=2$ and $7 \mathrm{~cm}$ ) starts to rise at first, and the temperature at the measuring points farther away (e.g., at $x=27$ and $42 \mathrm{~cm}$ ) also gradually increases due to the effect of heat conduction. When the temperature stabilizes, the difference in the temperature gradient between every two measuring points is obvious. During the subsequent cooling, the temperature at each measuring point decreases as the temperature near the heat source (i.e., the heating boundary; Figure 2) decreases and compared to the heating stage, the temperature gradient between the measuring points greatly decreases. During the second temperature cycle, the change process also reveals a similar law.

Figure 2 indicates that, during the first heating stage, the maximum temperature at measuring point $1(x=2 \mathrm{~cm})$ nearest to the heating end reaches $71.5^{\circ} \mathrm{C}$, while that during the second heating stage is $70.3^{\circ} \mathrm{C}$. After the first cooling step, the minimum temperature at this point is $26.5^{\circ} \mathrm{C}$, and the lowest temperature after the second cooling is $26.0^{\circ} \mathrm{C}$. It is observed that, under the action of cyclic temperature loading, the temperature of the column section away from 
TABLE 1: Test schemes for cyclic temperature loading.

\begin{tabular}{lccccc}
\hline Case & Dry density $\left(\mathrm{g} / \mathrm{cm}^{3}\right)$ & Volume moisture content $(\%)$ & Saturation & Placement direction & Heating end \\
\hline 1 & 1.4 & 25.2 & 0.557 & Horizontal & Left \\
2 & 1.4 & 25.2 & 0.557 & Vertical & Top \\
3 & 1.4 & 25.2 & 0.557 & Vertical & Bottom \\
4 & 1.3 & 23.4 & 0.476 & Vertical & Top \\
5 & 1.2 & 21.6 & 0.407 & Horizontal \\
\hline
\end{tabular}

TABLE 2: Test schemes for heavy metal transfer.

\begin{tabular}{lcccc}
\hline Case & Dry density $\left(\mathrm{g} / \mathrm{cm}^{3}\right)$ & Volume moisture content $(\%)$ & Saturation & Heavy metal contaminant \\
\hline 6 & 1.4 & 25.2 & 0.557 & $\mathrm{Cu}^{2+}$ \\
7 & 1.3 & 23.4 & 0.476 & $\mathrm{Cu}^{2+}$ \\
8 & 1.2 & 21.6 & 0.407 & $\mathrm{Cu}^{2+}$ \\
9 & 1.4 & 25.2 & 0.557 & $\mathrm{Cu}^{2+}$ \\
10 & 1.4 & 25.2 & 0.557 & $\mathrm{Cd}^{2+}$ \\
\hline
\end{tabular}

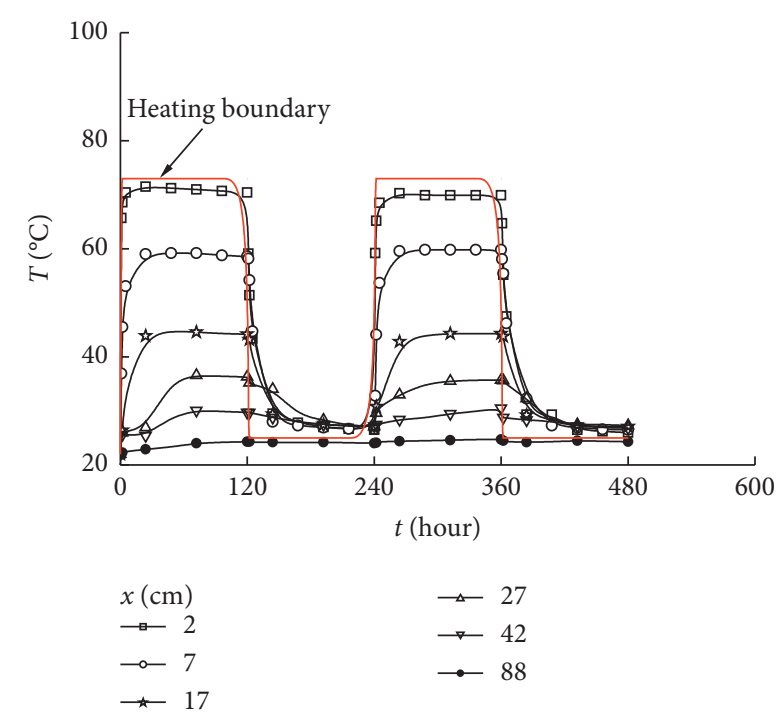

Figure 2: Temperature evolution processes over time for the two heating cycles.

the heat source (viz., at measuring point $8, x=88 \mathrm{~cm}$ ) is generally maintained at approximately $24.3^{\circ} \mathrm{C}\left( \pm 1^{\circ} \mathrm{C}\right)$, which indicates that the length of the soil column used in the tests suitably maintains a constant temperature at the other end (i.e., the low-temperature end).

On the whole, the other four cases (i.e., cases 2 to 5 in Table 1) also exhibit similar laws, but their magnitudes are quite different. In addition, the stable temperature values during the two cycles are different. The main difference is that after the second temperature cycle, the temperature at the measuring points near the higher-temperature end (at $x=2 \mathrm{~cm}$ ) is lower than that during the first cycle. In contrast, the temperature of the column section away from the heat source is low (at $x=42 \mathrm{~cm}$ ). This phenomenon shows that the cooling process during the first cycle imposes an obvious impact on the temperature evolution of the second heating process, which reflects the influence of the heating path. Now, certain factors are examined below, such as the dry density of the soil column and its placement direction.
Figure 3 shows the distribution curves along the heat conduction distance for the different dry densities and placement directions of the soil column during the first temperature cycle when the heating process has stabilized (i.e., at $t=120 \mathrm{~h}$ ). Figure 3(a) indicates that, for the same placement direction (e.g., cases 1 and 5 with the horizontal direction; cases 2 and 4 with the vertical direction and top heating; Table 1), the greater the dry density is, the larger the steady-state temperature at the same location of the soil column is. For example, the dry density in case 1 is set to $\rho_{\mathrm{d}}=1.4 \mathrm{~g} / \mathrm{cm}^{3}$ and that in case 5 is set to $\rho_{\mathrm{d}}=1.2 \mathrm{~g} / \mathrm{cm}^{3}$. At this time, the difference in the temperature distribution between these two cases can thus be clearly observed. However, the difference in temperature decreases for cases 2 and 4 because the difference in dry density between these two cases is slightly smaller (i.e., $\rho_{\mathrm{d}}=1.4$ and $1.3 \mathrm{~g} / \mathrm{cm}^{3}$ for cases 2 and 4, resp.). In fact, the dry density reflects the soil compactness. Therefore, the higher the soil dry density is, the larger the contact area between the soil particles is, and the thermal conductivity of soil with three-phase properties is closer to that of continuous solid particles [29, 31]; hence, the thermal conductivity increases with increasing dry density.

In fact, with increasing dry density, the soil column temperature rises faster (see Figure 3(a)). In addition, with increasing dry density, the specific heat capacity of soil increases with increasing moisture content $[29,33,38]$. This is closely related to the saturation degree of soils and also to the phase transition process between bound water and free water. As a result, more thermal energy is absorbed and subsequently released when the temperature stabilizes, which delays temperature stabilization. In other words, the heat conduction property and heat capacity are mutually restrained during the process of soil temperature stabilization [39]. For the different dry densities, it is generally considered that the heat conductivity is the fundamental factor influencing the temperature, while the impact of the specific heat capacity is relatively slight.

Figure 3(b) shows the influence of the placement direction of the soil column in the steady state, which indicates that the stable temperature value of the vertical soil column (cases 2 and 3) during heating is lower than that of the 

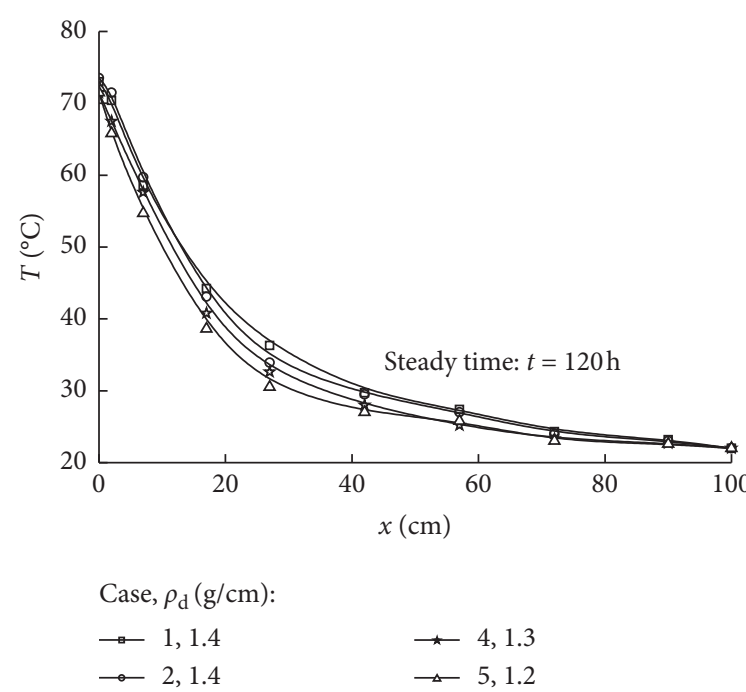

(a)
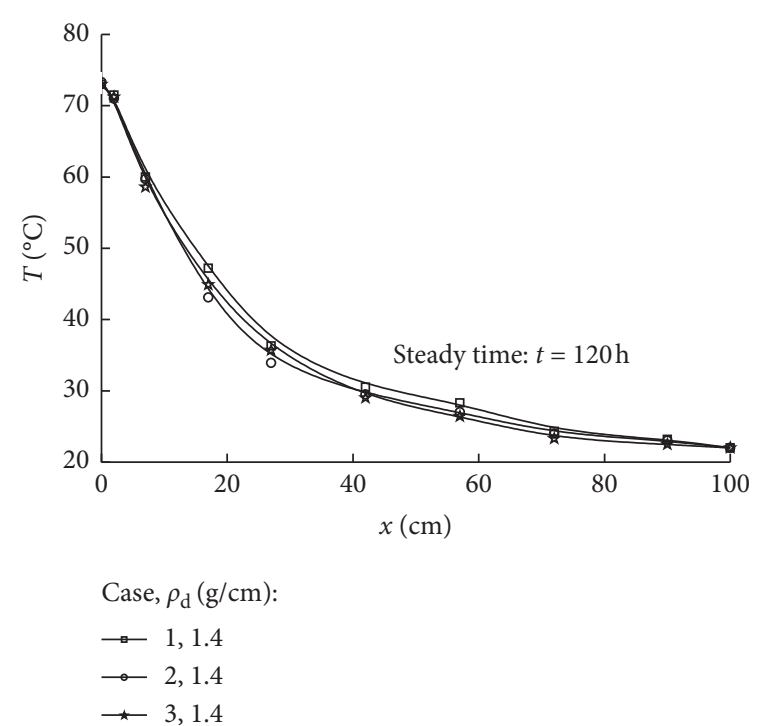

$\rightarrow \quad 3,1.4$

FIgURE 3: Steady-state temperature distribution along the distance: (a) effect of the dry density and (b) effect of the placement direction.

horizontally placed column (case 1). This is attributed to the effect of gravity, namely, for the vertical soil column with the heating end at the top, the amount of high-temperature vapor migrating to the low-temperature end is very small, while for the vertical soil column with the heating end at the bottom (case 3), the directions of the temperature and gravity potentials are opposite, and the amount of liquid water near the heat source is also small. When the soil column is horizontal (case 1), the influence of the gravity potential on moisture migration (including liquid and gaseous water) is weaker than that when the soil column is vertical. Moreover, the driving effect of the temperature potential leads to more moisture transferring heat from the heating end to the low-temperature end. Generally, compared to heat conduction, the influence of heat convection on the temperature distribution of unsaturated soils is limited [31].

3.2. Moisture Evolution Process. Under the action of the temperature gradient at both ends of the soil column, heat transfer will cause temperature redistribution in the unsaturated soil and then cause moisture migration in the soil pores. Figure 4 shows the evolution processes of moisture migration caused by the temperature over time under three different soil column placement direction conditions (cases 1 to 3). Figure 4 indicates that the moisture content at the measuring points close to the heat source (viz., at $x=2$ and $7 \mathrm{~cm}$ ) gradually decreases as the heating time increases. In the meantime, the moisture content away from the heat source (viz., at $x=57$ and $72 \mathrm{~cm}$ ) increases, resulting in an obvious reverse moisture content gradient. In contrast, when the temperature drops (i.e., during the cooling process), the moisture content at the points close to the cooling end (viz., at $x=2$ and $7 \mathrm{~cm}$ ) gradually increases. Nevertheless, at the points away from the cooling end (viz., at $x=57$ and $72 \mathrm{~cm}$ ), it gradually decreases. During the second temperature cycle, the moisture evolution also presents a similar phenomenon. For example, in case 1 (Figure $4(\mathrm{a})$ ), the point at $x=2 \mathrm{~cm}$ is the closest to the heating end. During the first temperature cycle, the moisture reduction caused by the temperature reaches $\Delta \theta=-4.9 \%$ in the steady state, and during the second temperature cycle, the moisture reduction is $\Delta \theta=-2.0 \%$. During the cooling stage, the moisture content at this point increases by $1.3 \%$ in the first temperature cycle and $0.5 \%$ in the second temperature cycle. In addition, the point at $x=88 \mathrm{~cm}$ is close to the low-constant-temperature end, and its moisture increase is $\Delta \theta=2.4 \%$ during the first cycle and $1.4 \%$ during the second cycle. The moisture change is $\Delta \theta=1.2 \%$ in the process of cooling stage in the first cycle and it is $\Delta \theta=-1.0 \%$ in the process of cooling in the second cycle.

Due to the difference in the dry density, the placement direction of the soil column, and the location of the heating boundary (top or bottom), the moisture distribution inside the soil column is also distinct (Figure 5), and the moisture distribution caused by the two temperature cycles also varies. Comparing the two cases (cases 1 and 5, or cases 2 and 4; Figure 5(b)) with the same placement direction of the soil column, it is found that when the dry density decreases, the change range of the moisture content increases along the soil column. This occurs because when the dry density is low, the soil porosity correspondingly increases, the soil is loose, and the connectivity between the particle pores is good.

However, the lower the soil dry density is, the lower the saturation degree is (please refer to Table 1). There are more open migration channels and larger storage spaces in the process of moisture migration, which eventually leads to a larger change in the moisture content.

Figure 5(a) also demonstrates that, in the case of the horizontal displacement direction (cases 1 and 5), the variation of moisture content in the soil column is generally 

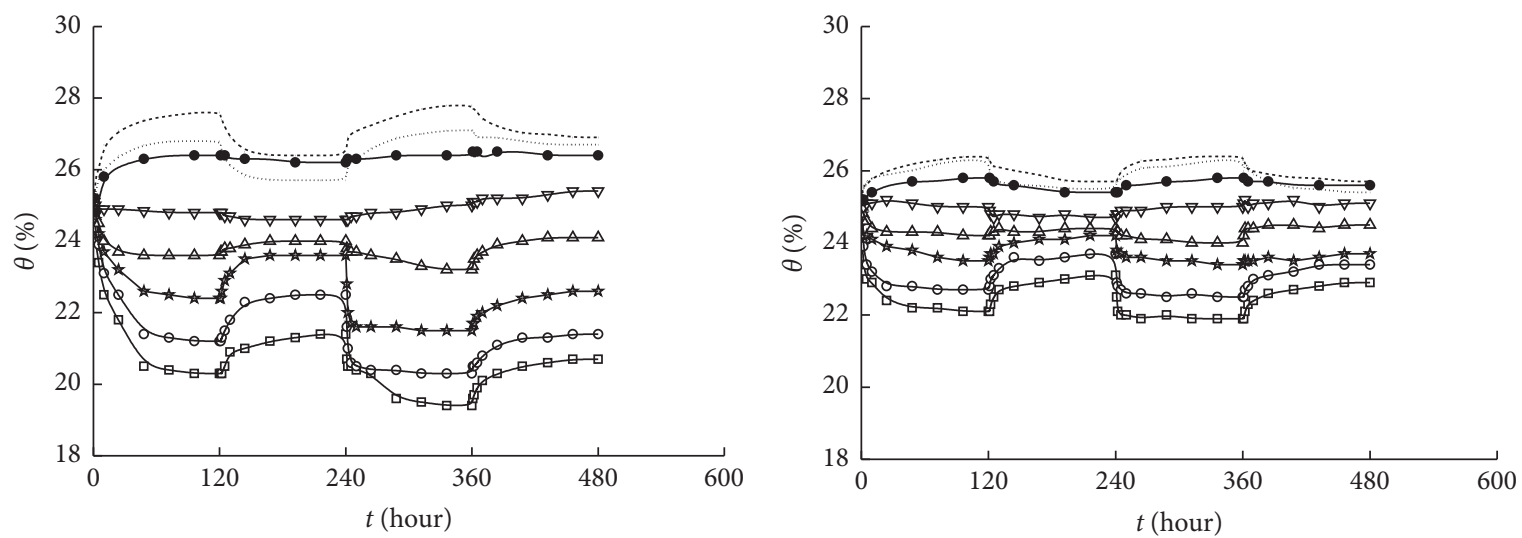

$\begin{array}{ll}x(\mathrm{~cm}) & \rightarrow 42 \\ \rightarrow-2 & \ldots 557 \\ \rightarrow 7 & --72 \\ \rightarrow 17 & \rightarrow-88 \\ \triangle 27 & \end{array}$

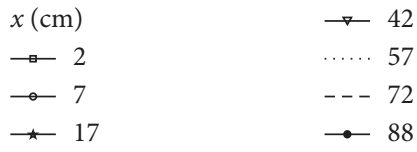

(a)

(b)
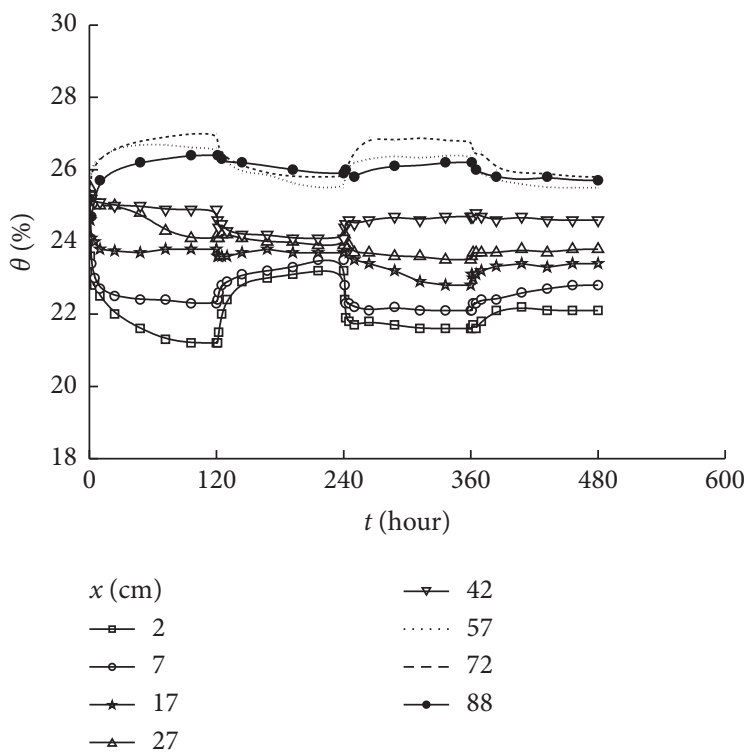

(c)

Figure 4: Moisture evolution processes over time for the two heating cycles: (a) horizontal placement, (b) vertical placement and top heating, and (c) vertical placement and bottom heating.

greater than that of the soil column placed vertically (cases 2 and 4; also see Figures 4(b) and 4(c)), and in addition, the effect of the gravity potential can be ignored (Figure 5(a)). When the soil column is vertical (cases 2 and 3 in Figure 5(b)), the soil moisture travels in the state of gaseous water and liquid free water under the action of the temperature potential. However, when the upper boundary temperature is higher than the lower boundary temperature (case 2), the directions of the temperature and gravity potentials are consistent, but due to the low density of gaseous water, in the soil near the heating end, less gaseous water and more liquid water move downward. However, when the temperature of the lower boundary is higher than that of the upper boundary (case 3), the direction of the temperature potential is the opposite to that of the gravity potential. As a result, less liquid water migrates due to gravity, but gaseous water notably migrates due to the thermal diffusion effect. Finally, the moisture content change in the soil column under vertical heating (cases 2 and 3; Figure 5(b)) is greater than that in the soil column under horizontal heating (case 1).

At the completeness of the heating stage, the gradient of the temperature inside the soil column greatly decreases due to the latter cooling process (Figure 6), and the effect of the solid matrix potential becomes prominent. When the soil column is placed horizontally, the influence of the gravity potential can be ignored. When the soil column is placed vertically and the top boundary temperature is higher than the lower boundary temperature, 

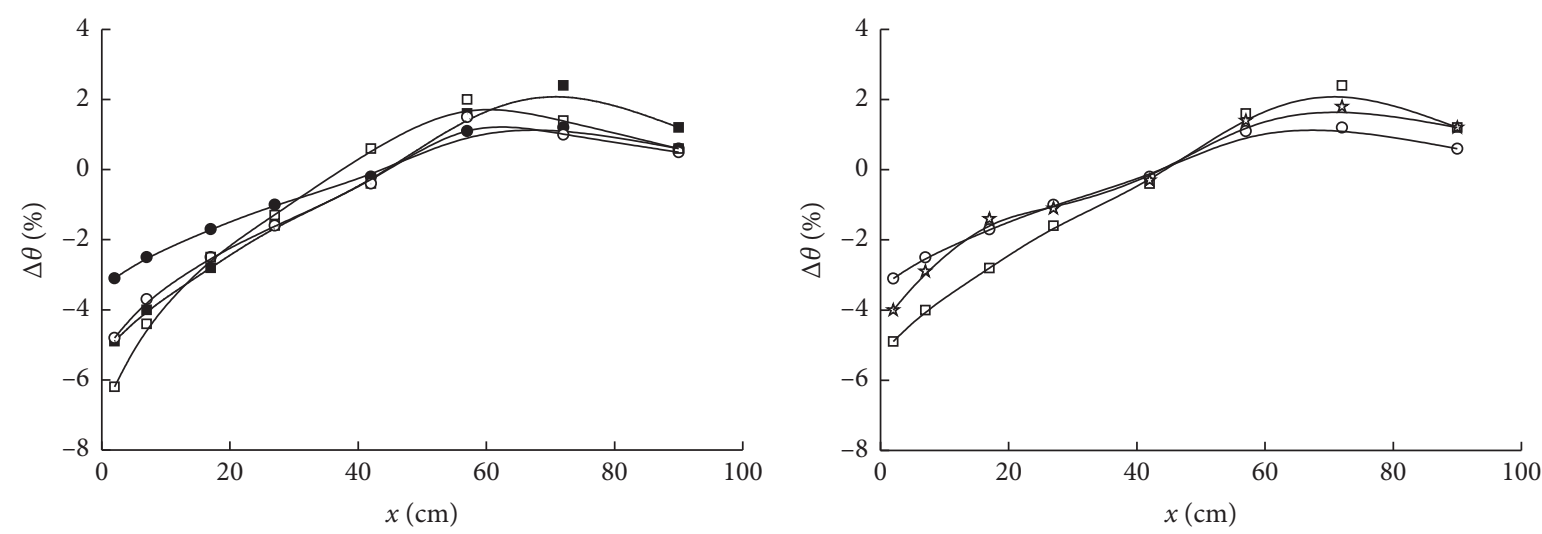

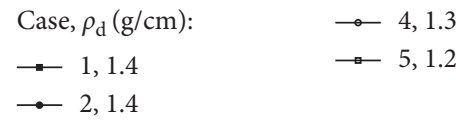

(a)

$$
\begin{aligned}
& \text { Case, } \rho_{\mathrm{d}}(\mathrm{g} / \mathrm{cm}) \text { : } \\
& -\quad 1,1.4 \\
& \rightarrow \quad 2,1.4 \\
& \rightarrow \quad 3,1.4
\end{aligned}
$$

(b)

FIGURE 5: The moisture content variations caused by heating in the different cases $(t=120 \mathrm{~h}$ ): (a) effect of the dry density and (b) effect of the placement direction.

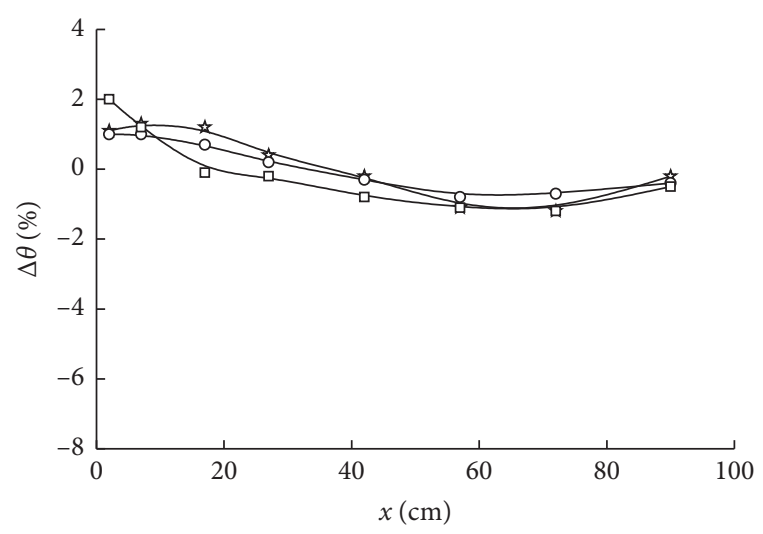

$$
\begin{aligned}
& \text { Case, } \rho_{d}(\mathrm{~g} / \mathrm{cm}) \text { : } \\
& \leftarrow \quad 1,1.4 \\
& \rightarrow 2,1.4 \\
& =3,1.4
\end{aligned}
$$

FIgURE 6: The moisture content variations caused by cooling in the different cases $(t=120 \mathrm{~h})$.

the moisture content gradient direction is upward (i.e., the matrix potential direction is upward), and the direction of the matrix potential is the opposite to that of the gravity potential. Moreover, less liquid water migrates. When the temperature at the lower boundary of the soil column is higher than the temperature at the upper boundary, the direction of the matrix potential caused by the moisture content gradient is consistent with that of the gravity potential, and the liquid water migration caused by gravity increases. In addition, a comparison of Figures 2 and $4(\mathrm{a})$ indicates that the change in moisture and the time required to reach stability lag behind the temperature.
3.3. Heavy Metal Pollutant Evolution Process. Figure 7 shows the concentration distributions of the two heavy metal ions $\left(\mathrm{Cu}^{2+}\right.$ and $\left.\mathrm{Cd}^{2+}\right)$ in the soil column after $240 \mathrm{~h}$ of temperature-driven action by monotonous heating on the left end of the soil column. These experiments consider different initial moisture contents, dry densities, and heavy metal ions (Table 2). The experimental results reveal that, without temperature-driven action (case 9; Figure 7(a)), the migration amount of $\mathrm{Cu}^{2+}$ is very small due to the possible molecular diffusion of heavy metal ions. The presence of a temperature gradient effectively promotes the transfer of heavy metal ions in the kaolin soil column (Figure 7(a), cases 6 to 8 ), which can be attributed to the significant convection of liquid free water in soil pores caused by the temperature variation and, of course, mechanical dispersion. Moreover, the maximum migration distance of the $\mathrm{Cu}^{2+}$ ions reaches $20 \mathrm{~cm}$, which indicates that the migration distance of $\mathrm{Cu}^{2+}$ increases due to the temperature-driven action. In fact, as the moisture in the soil column moves from high- to lowtemperature locations, the heavy metal ions in the pollution source dissolve in the liquid water and migrate from the heating end to the low-temperature end mainly with the liquid water movement. In addition, the movement of gaseous water hardly carries any heavy metal ions.

The higher the dry density is, the smaller the migration distance of the heavy metals is, which is related to the small pores and limited liquid water migration. In particular, the smaller the pores between the solid soil particles are, the less liquid water there is functioning as a carrier of the heavy metal ions, and the more unfavorable the convection and diffusion of heavy metals are. As mentioned above, the temperature gradient increases with increasing dry density, the proportion of liquid water to the total amount of transferred water is low, and the migration amount of the heavy metals is small. 


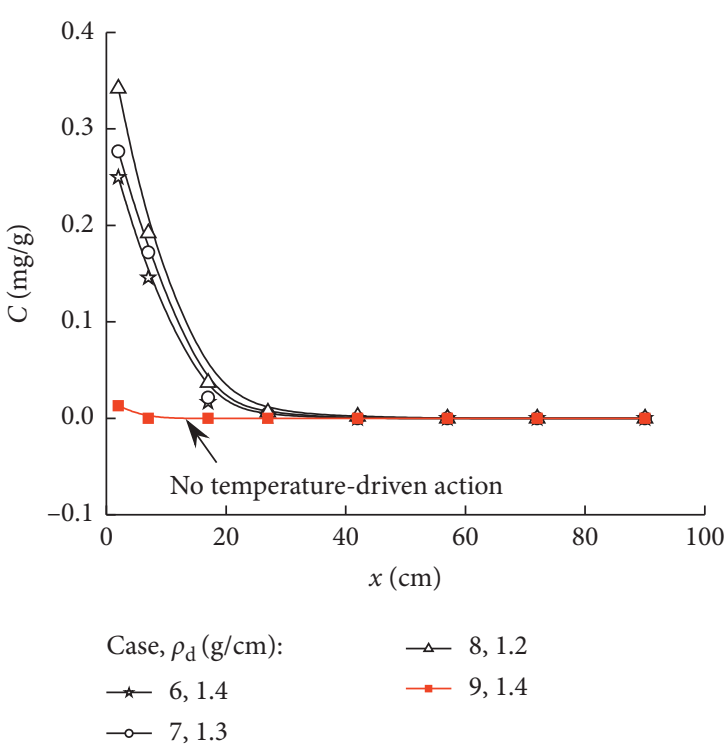

(a)

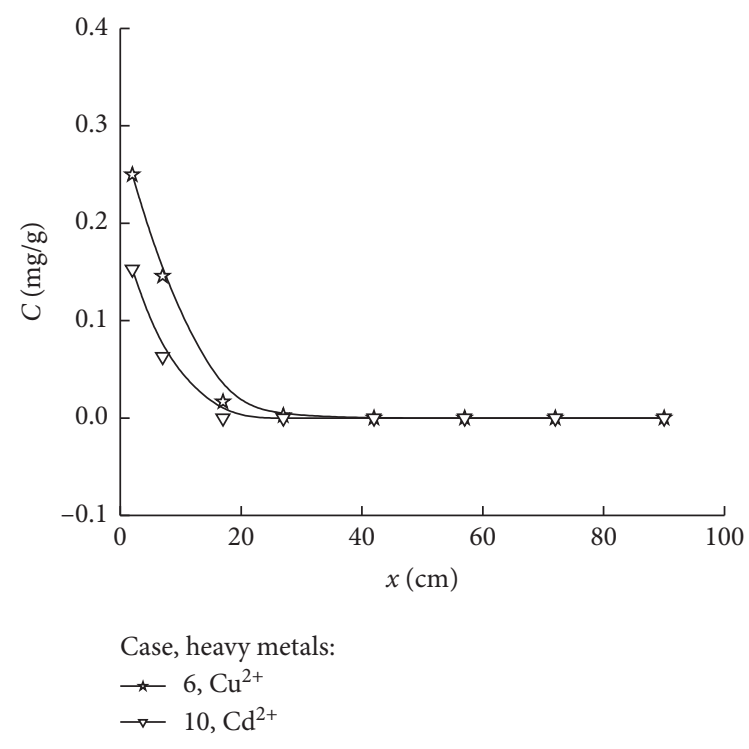

(b)

FIGURE 7: Concentration distributions of the heavy metal ions caused by the temperature: (a) effect of the dry density and (b) the different heavy metals.

Figure 7(b) also depicts a comparison of the migration amounts of $\mathrm{Cu}^{2+}$ and $\mathrm{Cd}^{2+}$ ions in the soil column. Moreover, the concentrations of the two heavy metal ions near the heating end are the same; namely, the concentrations of the pollutant source are both $1 \mathrm{mg} / \mathrm{g}$. The results show that the migration amount and distance of the two heavy metals are quite different due to the temperature-driven action. Because the adsorption capacity of $\mathrm{Cd}^{2+}$ ions to soil is much higher than that of $\mathrm{Cu}^{2+}$ ions, the migration amount and concentration distance of the $\mathrm{Cu}^{2+}$ ions are greater than those of the $\mathrm{Cd}^{2+}$ ions. Notably, the difference in adsorption mode between these two heavy metal ions leads to different migration rules. Certainly, the adsorption effect of heavy metal ions by soil particles is closely related to the type of soil. That is, the physicochemical interaction between soil particles and water on the migration process of heavy metal ions has an important impact, which is worthy of further discussion.

\section{Conclusions}

The physical mechanism of heat transfer, moisture migration, and heavy metal transfer in kaolin soils was studied by onedimensional column tests. Heat energy was mainly transferred in the form of heat conduction in the unsaturated soils, and moisture was transferred in the form of gaseous and liquid water. The change in moisture and time required to reach stability lagged behind the temperature change on the whole.

The cyclic temperature tests indicate that, during the second cycle, the temperature of the soil column near the heat source is lower than that during the first cycle. In contrast, the temperature of the soil column far away from the heat source is low, which reflects the influence of the heating path. Correspondingly, the moisture distribution during the second cycle is quite different from that during the first cycle due to the difference in the initial moisture content conditions between the two cycles.
The initial soil dry density, placement direction of the soil column, and set of temperature boundaries greatly affect heat conduction, moisture migration, and heavy metal transfer. The higher the dry density is, the better the heat transfer is. The lower the dry density is, the more favorable the moisture migration is. The placement direction of the soil column and the set of temperature boundaries influence the moisture distribution through the difference in the temperature, gravity, and solid matrix potentials.

Temperature-driven liquid water movement could effectively promote the transfer of heavy metals in kaolin soils, which is closely correlated with the convection of liquid free water. However, the transfer of heavy metal ions in soils induced by molecular diffusion is not obvious due to the absence of a thermal driving force. The higher the soil dry density is, the smaller the migration distance of the heavy metal pollutants is, which is related to the small pores and limited liquid water movement. In view of the obvious adsorption difference of copper ions $\left(\mathrm{Cu}^{2+}\right)$ and lead ions $\left(\mathrm{Cd}^{2+}\right)$, the transfer distance and deposited concentration values of $\mathrm{Cu}^{2+}$ are much greater than those of $\mathrm{Cd}^{2+}$.

\section{Data Availability}

The data used to support the findings of this study are available from the corresponding author upon request.

\section{Conflicts of Interest}

The authors declare that there are no conflicts of interest.

\section{Acknowledgments}

This work was supported by the National Natural Science Foundation of China (51979002). 


\section{References}

[1] Y. Wang, F. Chen, X. Li, X. Yin, and Y. Lei, "The variable-mass seepage law of broken porous rock: an experimental study," Geomatics, Natural Hazards and Risk, vol. 11, no. 1, pp. 1991-2005, 2020.

[2] C. Yu, R. Liao, X. Cai, and X. Yu, "Sodium polyacrylate modification method to improve the permeant performance of bentonite in chemical resistance," Journal of Cleaner Production, vol. 213, pp. 242-250, 2019.

[3] B. Bai and T. Li, "Irreversible consolidation problem of a saturated porothermoelastic spherical body with a spherical cavity," Applied Mathematical Modelling, vol. 37, no. 4, pp. 1973-1982, 2013.

[4] B. Bai, J. Wang, Z. Zhai, and T. Xu, "The penetration processes of red mud filtrate in a porous medium by seepage," Transport in Porous Media, vol. 117, no. 2, pp. 207-227, 2017.

[5] S. Lee, I.-W. Ko, I.-H. Yoon, D.-W. Kim, and K.-W. Kim, "Colloid mobilization and heavy metal transport in the sampling of soil solution from duckum soil in South Korea," Environmental Geochemistry and Health, vol. 41, no. 1, pp. 469-480, 2019.

[6] Y.-J. Du, M.-L. Wei, K. R. Reddy, Z.-P. Liu, and F. Jin, "Effect of acid rain $\mathrm{pH}$ on leaching behavior of cement stabilized lead-contaminated soil," Journal of Hazardous Materials, vol. 271, pp. 131-140, 2014.

[7] B. Bai, T. Xu, and Z. Guo, "An experimental and theoretical study of the seepage migration of suspended particles with different sizes," Hydrogeology Journal, vol. 24, no. 8, pp. 2063-2078, 2016.

[8] D. Rao and B. Bai, "Study of the factors influencing diffusive tortuosity based on pore-scale SPH simulation of granular soil," Transport in Porous Media, vol. 132, no. 2, pp. 333-353, 2020.

[9] S. Raikova, M. Piccini, M. K. Surman, M. J. Allen, and C. J. Chuck, "Making light work of heavy metal contamination: the potential for coupling bioremediation with bioenergy production," Journal of Chemical Technology \& Biotechnology, vol. 94, no. 10, pp. 3064-3072, 2019.

[10] R. Valsala and S. K. Govindarajan, "Co-colloidal BTEX and microbial transport in a saturated porous system: numerical modeling and sensitivity analysis," Transport in Porous Media, vol. 127, no. 2, pp. 269-294, 2019.

[11] B. Bai, J. Zhang, L. Liu, and Y. Ji, "The deposition characteristics of coupled lead ions and suspended silicon powders along the migration distance in water seepage," Transport in Porous Media, vol. 134, no. 2, pp. 1-18, 2020.

[12] B. Bai, D. Rao, T. Chang, and Z. Guo, "A nonlinear attachment-detachment model with adsorption hysteresis for suspension-colloidal transport in porous media," Journal of Hydrology, vol. 578, Article ID 124080, 2019.

[13] B. Abbar, A. Alem, A. Pantet et al., "Effect of natural geotextile on the cotransport of heavy metals $\left(\mathrm{Cu}^{2+}, \mathrm{Pb}^{2+}\right.$, and $\left.\mathrm{Zn}^{2+}\right)$ and kaolinite particles," Environmental Technology, vol. 42, no. 4, pp. 558-570, 2019.

[14] X. Chen and B. Bai, "Experimental investigation and modeling of particulate transportation and deposition in vertical and horizontal flows," Hydrogeology Journal, vol. 23, no. 2, pp. 365-375, 2015.

[15] B. Bai, F. Long, D. Rao, and T. Xu, "The effect of temperature on the seepage transport of suspended particles in a porous medium," Hydrological Processes, vol. 31, no. 2, pp. 382-393, 2017.
[16] X. Cui, Y. Fan, H. Wang, and S. Huang, "Experimental investigation of suspended particles transport in porous medium under variable temperatures," Hydrological Processes, vol. 33, no. 7, pp. 1117-1126, 2019.

[17] X. Chen, Z. Wu, Q. Cai, and W. Cao, "Effect of ultrasonic stimulation on particle transport and fate over different lengths of porous media," Journal of Hydrology, vol. 559, pp. 972-983, 2018.

[18] S. Taghipour, S. M. Hosseini, and B. Ataie-Ashtiani, "Engineering nanomaterials for water and wastewater treatment: review of classifications, properties and applications," New Journal of Chemistry, vol. 43, no. 21, pp. 7902-7927, 2019.

[19] K. Bär, W. Rühaak, B. Welsch, D. Schulte, S. Homuth, and I. Sass, "Seasonal high temperature heat storage with medium deep borehole heat exchangers," Energy Procedia, vol. 76, pp. 351-360, 2015.

[20] X. Cui, Q. Liu, C. Zhang, Y. Huang, Y. Fan, and H. Wang, "Land subsidence due to groundwater pumping and recharge: considering the particle-deposition effect in ground-source heat-pump engineering," Hydrogeology Journal, vol. 26, no. 3, pp. 789-802, 2018.

[21] M. H. E. Monged, M. T. Hussein, and A. E. M. Khater, "Elemental and radiological aspects of geothermal springs and nearby soil and sediment of Al-Lith area: concentration and risk assessment," Environmental Earth Sciences, vol. 77, no. 12, p. 427, 2018.

[22] C. Yu, M. Zhou, J. Ma, X. Cai, and D. Fang, “Application of the homotopy analysis method to multispecies reactive transport equations with general initial conditions," Hydrogeology Journal, vol. 27, no. 5, pp. 1779-1790, 2019.

[23] M. Funk and K. G. Wakili, "Driving potentials of heat and mass transport in porous building materials: a comparison between general linear, thermodynamic and micromechanical derivation schemes," Transport in Porous Media, vol. 72, no. 3, pp. 273-294, 2008.

[24] C. Miracapillo and L. Ferroni, "Numerical simulation of radionuclides migration in the far field of a geological repository," Energy Procedia, vol. 82, pp. 848-854, 2015.

[25] P. T. Nguyen and O. Amiri, "Study of the chloride transport in unsaturated concrete: highlighting of electrical double layer, temperature and hysteresis effects," Construction and Building Materials, vol. 122, pp. 284-293, 2016.

[26] A. M. Tang and Y. J. Cui, "Effects of mineralogy on thermohydro-mechanical parameters of MX80 bentonite," Journal of Rock Mechanics and Geotechnical Engineering, vol. 2, no. 1, pp. 91-96, 2010.

[27] H. A. Basha and A. P. S. Selvadurai, "Heat-induced moisture transport in the vicinity of a spherical heat source," International Journal for Numerical and Analytical Methods in Geomechanics, vol. 22, no. 12, p. 969, 1998.

[28] H. R. Thomas, Y. He, and C. Onofrei, "An examination of the validation of a model of the hydro/thermo/mechanical behaviour of engineered clay barriers," International Journal for Numerical and Analytical Methods in Geomechanics, vol. 22, no. 1, pp. 49-71, 1998.

[29] F. Lindner, M. Pfitzner, and C. Mundt, "Experimental and numerical study of multiphase, multicomponent flow in porous media with a multiphase mixture model," Transport in Porous Media, vol. 116, no. 1, pp. 143-161, 2017.

[30] B. Bai, D. Rao, T. Xu, and P. Chen, "SPH-FDM boundary for the analysis of thermal process in homogeneous media with a discontinuous interface," International Journal of Heat and Mass Transfer, vol. 117, pp. 517-526, 2018. 
[31] B. Bai, G.-C. Yang, T. Li, and G.-S. Yang, "A thermodynamic constitutive model with temperature effect based on particle rearrangement for geomaterials," Mechanics of Materials, vol. 139, Article ID 103180, 2019.

[32] X. Cui, Q. Liu, and C. Zhang, "Detachment characteristics of deposited particles in porous medium: experimentation and modeling," Transport in Porous Media, vol. 119, no. 3, pp. 633-647, 2017.

[33] B. Bai and X. Shi, "Experimental study on the consolidation of saturated silty clay subjected to cyclic thermal loading," Geomechanics and Engineering, vol. 12, no. 4, pp. 707-721, 2017.

[34] Y.-J. Du, J. Wu, Y.-L. Bo, and N.-J. Jiang, "Effects of acid rain on physical, mechanical and chemical properties of GGBSMgO-solidified/stabilized Pb-contaminated clayey soil," Acta Geotechnica, vol. 15, no. 4, pp. 923-932, 2020.

[35] N. Finzgar and D. Lestan, "Advanced oxidation for treatment of aqueous extracts from EDTA extraction of $\mathrm{Pb}$ and $\mathrm{Zn}$ contaminated soil," Journal of Environmental Engineering, vol. 132, no. 10, pp. 1376-1380, 2006.

[36] P. Zhang, B. Bai, S. Jiang, P. Wang, and H. Li, “Transport and deposition of suspended particles in saturated porous media: effect of hydrodynamic forces and pore structure," Water Supply, vol. 16, no. 4, pp. 951-960, 2016.

[37] G. C. Yang and B. Bai, "Thermo-hydro-mechanical model for unsaturated clay soils based on granular solid hydrodynamics theory," International Journal of Geomechanics, vol. 19, no. 10, Article ID 04019115, 2019.

[38] Y. Wang, Z. Li, H. Jing, Y. Li, and M. Wang, "Study on the seepage characteristics of deep buried tunnels under variable high-pressure water heads," Bulletin of Engineering Geology and the Environment, vol. 80, no. 2, pp. 1477-1487, 2021.

[39] B. Bai, "Fluctuation responses of saturated porous media subjected to cyclic thermal loading," Computers and Geotechnics, vol. 33, no. 8, pp. 396-403, 2006. 